\title{
Errata do artigo "SOBRE A IMPORTÂNCIA DA TRANSCRIÇÃO FONÉTICA EM SISTEMAS DE RECONHECIMENTO DE FALA"
}

\author{
Carlos Alberto Ynoguti e Fábio Violaro \\ DECOM-FEEC-UNICAMP, Caixa Postal 6101, 13083-970 Campinas, SP, Brasil \\ fabio@decom.fee.unicamp.br
}

No artigo acima [1], devido ao problema de ausência de fontes IPA na edição final, resultaram erradas a Tabela 1 e as transcrições fonéticas em 4.2 , que são reapresentadas a seguir.

\begin{tabular}{|c|c|c|c|}
\hline Fone & Exemplo & Fone & Exemplo \\
\hline$\#$ & silêncio & $\mathrm{g}$ & $g$ orila \\
\hline $\mathrm{a}$ & $a$ çafrão & 3 & j iló \\
\hline $\mathrm{e}$ & e levador & $\mathrm{k}$ & $c$ achoeira \\
\hline$\varepsilon$ & pele & 1 & $l$ eão \\
\hline $\mathrm{i}$ & sino & $\kappa$ & Ih ama \\
\hline $\mathrm{j}$ & fu $i$ & $\mathrm{~m}$ & $m$ ontanha \\
\hline 0 & bo lo & $\mathrm{n}$ & $n$ évoa \\
\hline o & bo la & $j i$ & inthane \\
\hline $\mathrm{u}$ & $1 u \mathrm{a}$ & $\mathrm{p}$ & $p$ oente \\
\hline$\tilde{\alpha}$ & $\operatorname{maç} \tilde{a}$ & $\mathrm{r}$ & cer a \\
\hline$\tilde{\mathrm{e}}$ & sen ta & $\overline{\mathbf{r}}$ & cerr ado \\
\hline$\tilde{\mathrm{i}}$ & $\mathrm{p}$ in to & $\mathrm{R}$ & ca $r$ ta \\
\hline$\widetilde{o}$ & s om bra & s & $s$ apo \\
\hline$\tilde{\mathrm{u}}$ & um & $\mathfrak{t}$ & $t$ empes $t$ ade \\
\hline $\mathrm{b}$ & $b$ ela & ts & t igela \\
\hline$d$ & $d$ ádiva & $\mathrm{v}$ & $v$ erão \\
\hline dz & $d$ iferente & $\int$ & ch ave \\
\hline f & $f$ eira & $z$ & $z$ abumba \\
\hline
\end{tabular}

Tabell 1: Fones independentes de contexto, com seus respectivos exemplos.

\subsection{TRANSCRIÇÕES FONÉTICAS}

Como uma ilustração do quão diferente pode ser a pronúncia de uma mesma frase em função do sotaque dos locutores, são mostradas algumas transcrições fonéticas retiradas da base de dados para a frase: "O grêmio ganhou a quadra de esportes":

ogremjug $\widetilde{\alpha}$ nouakuadradeesportes

ugremjug $\widetilde{\alpha}$ nouakuadradzjsport $\int \mathrm{j} s$

ugremjug $\widetilde{\alpha}$ nou akuadradzj $\int p \circ \bar{r} t \int j \int$

\section{REFERENACIA}

[1] Carlos Alberto Ynoguti e Fábio Violaro, "Sobre a Importância da Transcrição Fonética em Sistemas de Reconhecimento de Fala", Revista da Sociedade Brasileira de Telecomunicações, Vol. 15, No. 2, pp. 44-49, junho 2000 . 